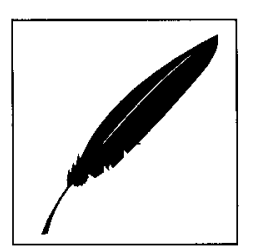

\section{Redat 3.0}

Mehr Komfort und Möglichkeiten mit der neuen Rechtsprechungsdatei

\section{Malte de Grabl}

Das neue Redat 3.0 übertrifft alle Erwartungen, die an eine Rechtsprechungsdatei gestellt werden. War schon die Vorversion wegen des klaren Aufbaus und der einfachen und schnellen Handhabung ein unentbehrlicher Helfer bei der täglichen Arbeit des Richters und Rechtsanwalts geworden, so hat sich Redat $3.0 \mathrm{zu}$ einer komfortablen leistungsstarken Datenbank entwickelt, die kaum noch Wünsche offenläßt.

\section{Anwendungsmöglichkeiten}

Welcher Jurist kennt bei der Bearbeitung eines Falles nicht die Situation, vor kurzem die maßgebliche Entscheidung dazu gelesen und vielleicht sogar abgelichtet zu haben? Doch wo war sie abgedruckt? Wo ist die Ablichtung geblieben? Ein Wiederfinden ist meist mit erheblichem Zeitaufwand verbunden, nicht selten auch aussichtslos. Hier ist Redat 3.0 von großem Nutzen. Es sagt ihnen sofort, wo sie sie abgelegt haben oder wiederfinden können. Das Suchen geht schnell und ist denkbar einfach. Dank der jederzeitigen Anzeige der möglichen Suchworte kann die Entscheidung sogar bei fehlerhafter Eingabe wiedergefunden werden.

\section{Persönliche Datenbank}

sönliche Datenbank auf dem eigenen PC am Schreibtisch des Richters oder des Rechtsanwalts. Der Zugriff kann jederzeit erfolgen. Die Auswahl der Daten ist schneller und leichter möglich. Die Übersicht geht nicht verloren, da der Anwender für seinen speziellen Bedarf die Auswahl trifft und deshalb der Umfang naturgemäß auf das Wesentliche beschränkt bleibt. Zudem sollen niicht primär unbekannte Entscheidungen gesucht, zeitaufwendig durchgesehen, sortiert und erstmals erarbeitet werden, sondern das eigentlich vorhandene Wissen soll schneller und vor allen Dingen sicherer wieder zur Verfügung stehen. Das tägliche Handwerkszeug wird durch Redat 3.0 schnell und einfach verwaltet und steht insbesondere an jedem Ort mit einem PC ohne Platz- und Transportprobleme zur Verfügung. Das erscheint mir ein wesentliches Argument gerade für Rechtsanwälte zu sein, besonders dann, wenn sie Rechtsprechung von spezialisierten Spruchkörpern sammeln. Der Zeitaufwand für die Eingabe wird durch die späteren Zeitvorteile und die Verbesserung der Arbeitsqualität mehr als ausgeglichen.

\section{Aktueller als jede Zeitschrift}

Und noch einen weiteren großen Vorteil hat Redat 3.0 im Vergleich zu Zeitschriften, juris oder einer CD-ROM, wie beispielsweise die Verhältnisse in Hamburg zeigen: Jeder Richter, der es wünscht, wird in der Hamburger Justiz mit Redat ausgestattet. Inzwischen sind es mehr als 50 Richter. Viele Kammern und Senate sind dazu übergegangen, aktuelle Entscheidungen, die nicht oder noch nicht veröffentlicht werden, einzugeben und dann an interessierte Kollegen weiterzuleiten. Die Rechtsprechung innerhalb der Gerichte wird dadurch aktueller und transparenter. Dies gilt nicht nur für die horizontale Ebene, sondern erst recht für die nachgeordneten Instanzen. So sind in Hamburg bereits umfangreiche Dateien zum Wohnraummietrecht, zum Straf- und Strafverfahrensrecht und zum Handelsrecht entstanden. Weitere $\mathrm{Da}$ teien zum Seehandelsrecht, zum Transportrecht und zum Gesellschaftsrecht sind im Aufbau begriffen.

Dank der mannigfaltigen Druckmöglichkeiten kann auch derjenige, der keinen PC an seinem Arbeitsplatz hat, die gesammelten Daten -. wie bei einem guten Nachschlagewerk üblich - nutzen. Neben der Loseblattsammlung kann ein Stichwort- und Paragraphenverzeichnis gedruckt werden, ein Vorteil übrigens, den die fertigen großen Datenbanken nicht bieten können. Nur nebenbei sei erwähnt, daß mit Redat 3.0 jetzt auch in den Bibliotheken problemlos gearbeitet werden kann, da bei der Installation die Möglichkeit gegeben ist, einen Änderungs- und Kopierschutz einzuschalten.

\section{Erweiterte Anwendungsmöglichkeiten}

Damit sind aber die Anwendungsmöglichkeiten nicht annähernd, rollständig beschrieken. Mag die Sammlung von Rechtsprechung von dem Verfasser Dr. Klaus Rühle auch vorrangig angestrebt worden sein, so hat Redat 3.0 den Kreis der möglichen Anwerĩder deutlich erweitert. Jetzt können problemlos zum Beispiel auch Verordnungen, dienstliche Anweisungen, Literatur, Definitiónen und ähn- 
liches mehr gesammelt und jederzeit wieder aufgerufen werden. Aber auch für die Karteikartensammlung des Studenten und das Lernen an der Universität kann Redat jetzt erfolgreich eingesetzt werden. Schließlich ist dieses einfach und schnell zu handhabende Programm auch für die private Katalogisierung von Sammlungen bestens geeignet.

\section{Wie arbeitet Redat 3.0?}

Nach dem Aufruf erscheint auf dem Bildschirm das Hauptmenü (Abb. 1).

Erkennbar wird, daß die persönliche Dateneingabe an erster Stelle steht und bereits unterlegt ist. Mit < Return $>$ werden die Eingabefelder geöffnet (Abb. 2).

Der Bildschirm für die Eingaben ist klar, übersichtlich geordnet und vollständig. Redat 3.0 bietet jetzt den unschätzbaren Vorteil, daß der Anwender jederzeit in fast jedem Feld über die <F2>Taste kontrollieren kann, ob er die Abkürzung oder die Schreibweise - so wie zuvor oder mit seinen Kollegen abgestimmt gleichlautend nutzt. Insbesondere kann er sofort feststellen, ob das Dokument bereits vorhanden ist. Nach dem Abspeichern der Daten wird dann gesondert der Text eingegeben. Dabei ist es ohne Schwierigkeiten möglich, einen Text, der sich bereits im ASCII-Format auf einem Datenträger befindet, $\mathrm{zu}$ übernehmen oder später in ein Textverarbeitungsprogramm zu exportieren, soweit dort ASCII-Zeichen verarbeitet werden können. In diesem Zusammenhang sei auch erwähnt, daß durchaus nicht jede Entscheidung selbst eingegeben werden muß, wenn mehrere Anwender gleichgerichtet „sammeln". So findet in Hamburg bereits ein reger Datenaustausch statt. Das Handbuch gibt hierfür zahlreiche Tips. Der Menüpunkt "Übertragung von/auf Diskette" ist sehr viel komfortabler geworden. Er gestattet das Anhängen an bereits vorhandene Datensät-
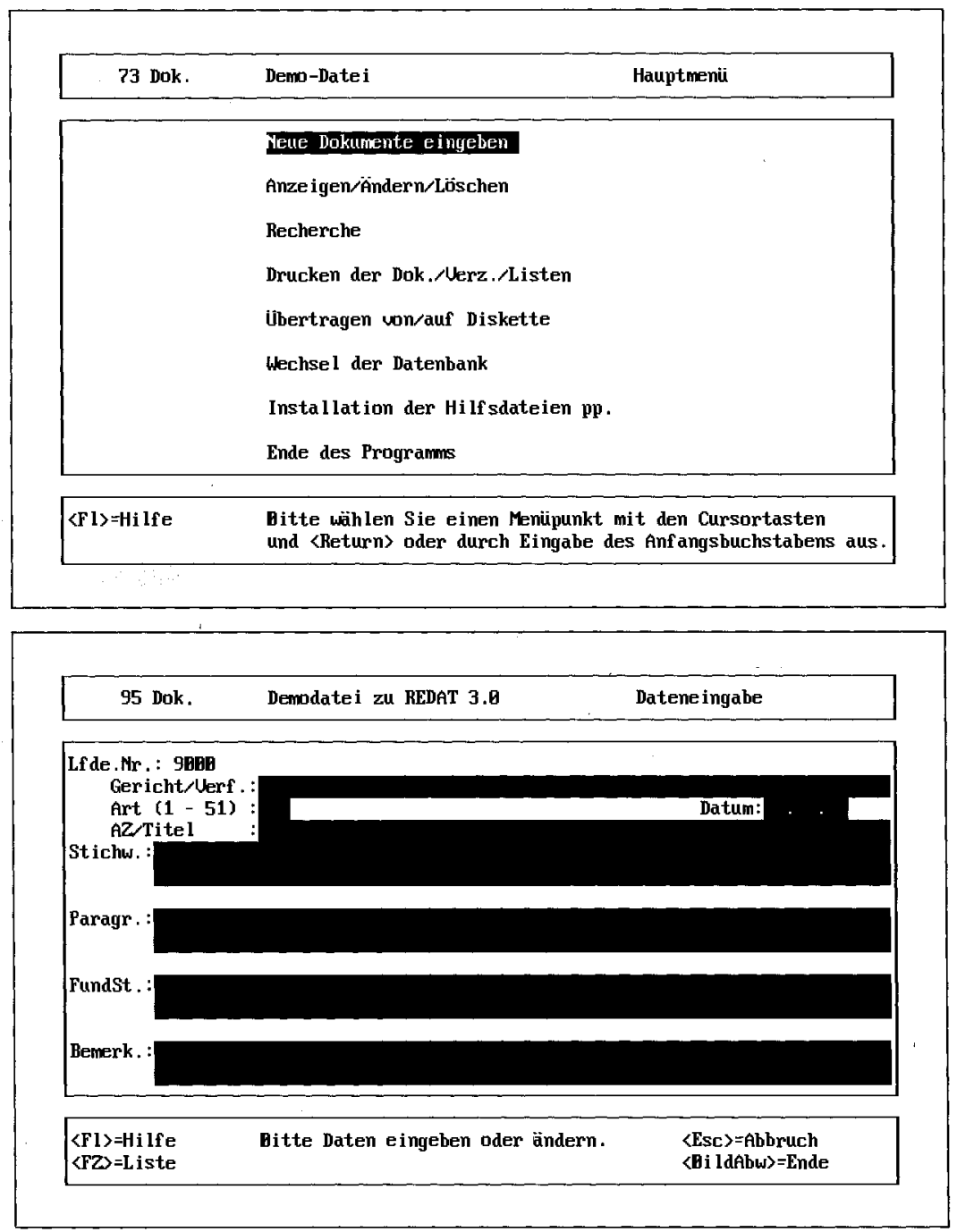

Abb. 1:

Das Hauptmenü

Abb. 2:

Die Eingabefelder

ze und ihren Aufruf, ohne daß sie bereits initialisiert worden sind. So kann man rechtzeitig entscheiden, ob man das Dokument übernehmen möchte.

\section{Die Suchfunktion}

Zweifellos liegt die besondere Stärke von Redat 3.0 in der umfangreichen, schnellen und zielsicheren Suchfunktion. Das Finden von Daten geschieht vorrangig über den Menüpunkt „Recherche”. Aber auch über „Anzeigen/Ändern/Löșchen” oder über die umfangreiche Sortierfunktion zur Vorbereitung des Ausdrucks ist ein schnelles Auffinden möglich.

Abbildung 3 (s. Folgeseite) zeigt, daß die Recherche denkbar einfach ist. Gesucht werden kann nach Stichworten, Paragraphen und Gericht. Wie bei den großen juristischen Datenbanken kön- nen die üblichen Verknüpfungen „und"/,oder" durchgeführt werden. Als besondere Unterstützung steht auch hier wieder die $<$ F2 $>$-Taste zur Verfügung, mit Hilfe derer die richtige und vollständige Schreibweise oder Formulierung gefunden und auch gleich übernommen werden kann. Zusätzlich kann jetzt noch die Hilfe einer Synonymdatei in Anspruch genommen werden, die den Benutzer sofort zu dem einschlägigen Stichwort führt.

Eine Volltextrecherche ist nicht vorgesehen. Hierin sehe ich insbesondere wegen der jederzeit über die <F2>-Taste Abb; 4 s. Folgeseite) zur Verfügung stehenden Stichwortliste keinen Nachteil. Nach meiner Erfahrung führt zwar die Recherche in Langtexten meist $\mathrm{zu}$ einer Vielzahl von „Treffern”, von denen dann aber oft nur wenige einschlägig sind. Zum gewünschten Ergebnis kommt.man erst nach zeitaufwendigem Lesen. Auch

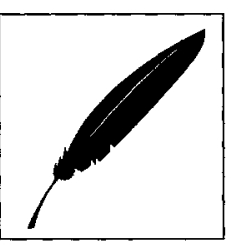

\section{(1)

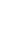



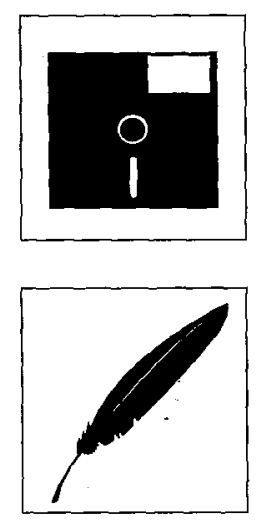

Abb.3:

Die Recherche

Abb.

Die Unterstüt $<F 2>-$ Taste

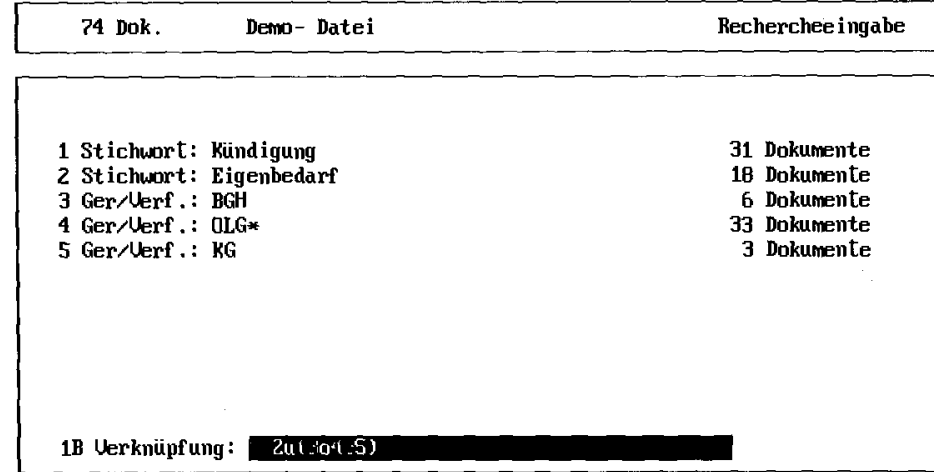

1B Uerknupfung :
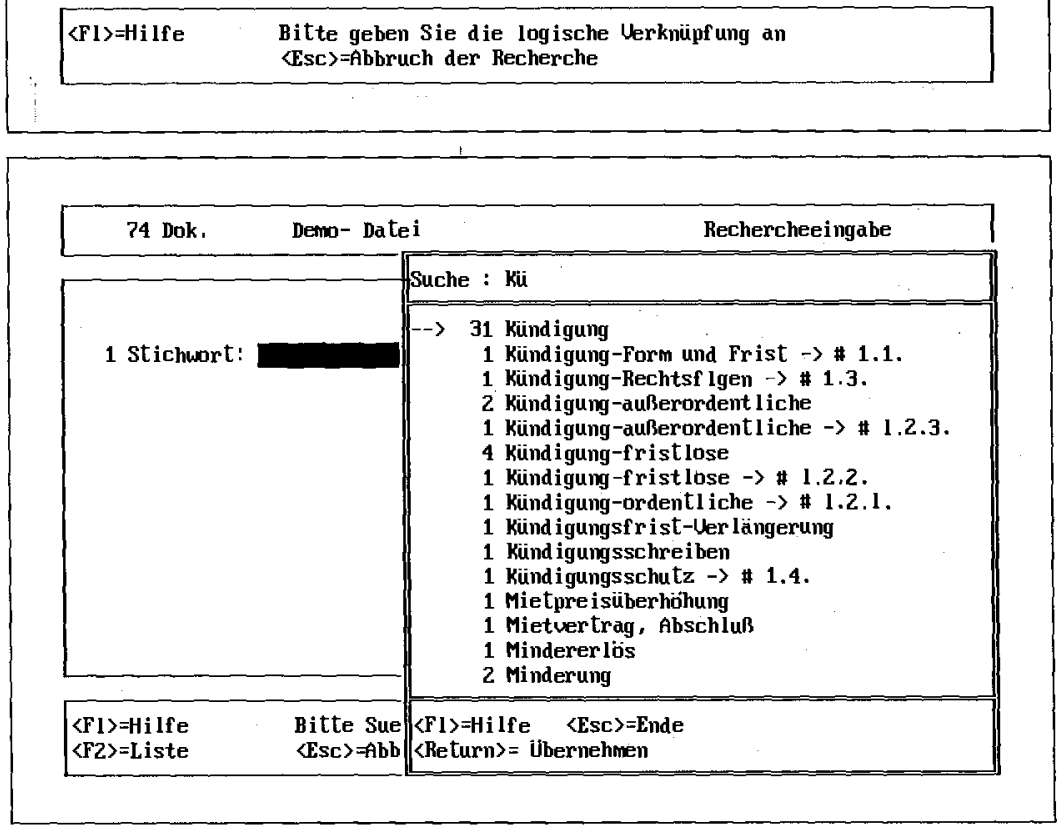

nimmt die Sammlung von Langtexten rasch erheblichen Speicherplatz in Anspruch. Zudem ist ein Dokumentenaustausch nicht mehr so problemlos möglich.

\section{Technische Daten}

Redat 3.0 läuft auf jedem IBMkompatiblen PC unter den Betriebssystemen ab MS-DOS 3.1 beziehungsweise DR-DOS. Das Programm kann bis zu 15 verschiedene Datenbanken verwalten. Die Anzahl der Datensätze pro Datenbank ist mit 9.000 reichlich bemessen. Pro Dokument können bis zu 64.000 Zeichen Text gespeichert werden. Das ist weit mehr, als man normalerweise braucht. Für Stichworte und Paragraphen stehen je 140 Zeichen pro Dokument zur Verfügung. Die Datensätze werden im dBASE-Format verwaltet.

\section{Installation}

Die Installation ist mit Hilfe des Setup-Programms einfach durchzuführen. Die Farb- und Drukkeranpassung hat den Standard von sehr viel teureren und größeren Programmen. Das Handbuch ist übersichtlich und klar gegliedert. Man braucht es nur zur Erstinstallation oder um einige Feinheiten beziehungsweise $\mathrm{Zu}$ sammenhänge nachzuschlagen, denn der klare Aufbau der Bildschirmmasken mit den zusätzlichen Hinweisen ist für die normale Anwendung bereits ausreichend. Zusätzlich stehen über die $<$ F1 $>$-Taste umfangreiche Hilfen zur Verfügung. Auch sind professionelle Sicherheiten gegen Fehler oder den Verlust von $\mathrm{Da}$ ten eingebaut worden.

\section{Zusammenfassung}

Zusammenfassend läßt sich sagen, daß hier ein Programm vorliegt, das bei einem Preis von lediglich 328,- DM umfangreiche Leistungen und eine ausgesprochen bequeme Handhabung bietet, die sonst nur bei wesentlich teureren Programmen zu finden sind. Es ist aus der Praxis für die Praxis geschaffen worden und besonders geeignet für den schlichten Anwender, der schnell und einfach zum Ziel kommen möchte. Dies wird erreicht durch eine klare, logische und doch zugleich komfortable Bedienung. Redat 3.0 ist ein ausgereiftes $\mathrm{Da}$ tenbankprogramm für den persönlichen Gebrauch am eigenen Schreibtisch, das nicht nur für den Richter und Rechtsanwalt geeignet ist, sondern für einen sehr viel größeren Kreis sehr schnell zu einem unentbehrlichen Helfer wird.

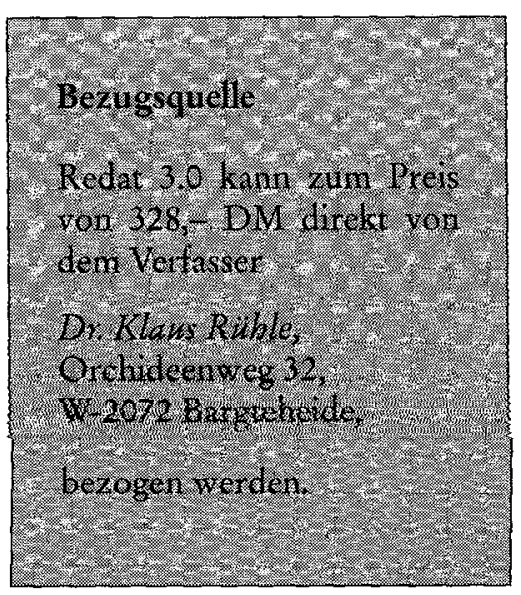

